\title{
Suggesting time frequency representation of the obtained reaction time of the consecutive tasks in a driving simulator for determining the rate of change of brain's frequency
}

\section{Prashant Rajdeep}

Baroda Medical College, The Maharaja Sayajirao University of Baroda

Lajja Patel ( $\sim$ lajja.patel-physiology@msubaroda.ac.in )

Baroda Medical College, The Maharaja Sayajirao University of Baroda

\section{Steffy CD}

Baroda Medical College, The Maharaja Sayajirao University of Baroda

Preeti Panchal

Baroda Medical College, The Maharaja Sayajirao University of Baroda

\section{Article}

Keywords: Reaction time (RT); Cognition; Driving simulator, Time frequency distribution (TFD), Rate of change of brain's frequency (ROCOF), Classification of driver

Posted Date: May 5th, 2022

DOI: https://doi.org/10.21203/rs.3.rs-1369242/v2

License: (1) This work is licensed under a Creative Commons Attribution 4.0 International License.

Read Full License 


\section{Abstract}

Objective - Suggesting an approach to determine the rate of change of brain's frequency (ROCOF).

Background - Reaction time (RT) is mostly well estimated in any driving simulator, especially the visual reaction time. However, an appropriate representation of RT in a driving simulator can help determine the changes in brain's frequency.

Method - We propose, use a driving simulator which can efficiently measure and record the participant's reaction times for the rapidly successive tasks in a simulator. A reciprocal presentation of each RT can be used as the brain's frequency for performing that task. Using this concept, a simulator software can calculate the brain's frequencies for all the tasks. While its graphical representation over time can yield time frequency distribution (TFD).

Results - Analysis of this time frequency representation (TDR) of RT may help to study the pattern of brain activity and to derive ROCOF, which can be used to classify the drivers.

\section{Introduction}

The human brain is an intricate structure, and understanding it is an intriguing task. The functions of brain cannot be clearly delineated; however, it has been proven to control the motor, sensory, autonomic functions along with speech, emotions, cognition, and homeostasis of the body. Apparently, most of the studies regarding these functions of brain consists of cognitive science. Cognition involves an interrelation of multiple mental processes and therefore requires a thorough understanding. Determining the speed of these mental processes is the essence of mental chronometry. Numerous studies have depicted reaction time (RT) as the valuable tool for measuring mental chronometry ${ }^{1}$. RT can be measured as the minimum time between the stimulus and the reaction that is obtained due to the stimulus. According to Oswal A et al. RT intimates about the probability of a stimulus estimated by the brain ${ }^{2}$. Likewise, accurate estimation of RT and its proper representation may help in estimating an interesting parameter like the 'rate of change of brain's frequency' (ROCOF) which in turn may help in quantifying the swiftness of action required in preventing mishaps.

\section{Material \& Methods}

RT is mostly well estimated in any driving simulator, especially visual reaction time. However, we generally measure RT for a particular task. But surprisingly, it varies, each time it is measured. Though this variation is marginal. An attempt should be made to check the dependency of this variation. To do so, a driving simulator which can efficiently measure and record the participant's reaction times for the rapidly successive tasks in the simulator can be used. For example, as done in the website 'http://physicsiology.com' where a simple visual simulation test has been performed, in which, the appearance of a red square or a circle on the monitor screen initiate the test, which is to be clicked with agility until the color of it turns green to terminate the test. ${ }^{3}$ It requires twenty-four clicks to complete the 
test, and the rate at which the participants responded, determines the result. The website compute by sorting all these reaction times in increasing order for its application. However, any advanced graphics can be used according to the selected simulator. As deriving RT by computer simulator remains the preferred one due to its ease, affordability, and compliance. Furthermore, experiments conducted by C. Ariën et al. and A. Akbari et al. suggest that though the traditional approach of obtaining real-time data of driving on the road is irreplaceable, it has many constraints which can be overcome by using surrogate techniques like a simulator that is close to various traffic environments and can provide with eventful data. $^{4,5}$

The above concept can also be understood by switch pattern. According to Schneider DW et al. and many others, the performance of one task after the other or same task repeatedly is the switch pattern. ${ }^{67}$ (Sinha et al., 2006) Based on which, the proposed switching pattern can be understood from the Fig. 1.

"Figure 1. Switching pattern". Example. Suppose a driving simulator consist of an event of driving a vehicle from point $A$ to point $B$, which consist of multiple tasks like task1- manoeuvrings of a steering after onscreen appearance of turn, task2- tackling speed breaker and so on (as depicted by different colour for each task). The simulator records these rapidly successive tasks. Hence.

On - refers to the appearance of an image on the screen for a task.

Off - refers to the manoeuvrings done (or simply the click)

Between On-Off -refers to the RT for the completion of the task.

Between Off \& On -refers to the processing speed of the computer processor, as well as the brain to select the next image for processing.

Numbers indicate the observed reaction time

\subsection{Data acquisition.}

After obtaining the RT for each task, the processing speed (in $\mathrm{Hz}$ ) of the brain for a particular task can be determined by reciprocating the RT. Similarly, the processing speed of the brain for consecutive tasks can be obtained in a dedicated simulator software.

\section{Results}

Plotting this data against the time (marking the respective frequency on the time frame when the response is recorded) can yield time frequency distribution (TFD) of the responses as shown in Fig. 2.

From the above derivation, an interesting parameter that is proposed is the ROCOF, i.e., the rate at which the processor shifts either slow to fast or reverse of it. 
ROCOF $=$ Difference in frequency between any two tasks/Duration between them

This parameter is inspired by the experimentation of task switching done by N. Sinha et al. (Sinha et al., 2006) Since, the efficiency of the brain to deal with the transition between different tasks, determines the incident of the crash. Therefore, the ROCOF from slow to fast or vice versa, can be an important parameter for predicting probability of driver to commit mistakes. Moreover, as also evident from 'switch cost' experimental model of D.W. Schneider et al., the compatibility of a driver is not only based on the RT for a particular event but also on the rapidity to switch from one task to another (Schneider \& Logan, 2005)

\section{Discussion}

Traditionally, RT is measured as a window to assess the cognitive function of the brain. Yet, it can have multiple possible applications, which may help yield new gateways for primeval processes.

Based on our proposal, the processing speed (in $\mathrm{Hz}$ ) of the brain can be determined by reciprocating the RT. However, the actual processing speed would be better than this if, we considered the complex analysis by taking the neurons and their synapses as an individual biological central processing unit (CPU), which run in parallel. ${ }^{8}$ While, in this case we have assumed the simplified analysis in which a group of neurons in a specific brain area is considered as a single CPU.

Diagnosis made based on pattern of symptoms, and their timely detection to forecast the onset of disease and its remedial measure shows the real expertise of a physician. Akin to this, we propose a system, that can determine the speed of the brain using RT and compute the ROCOF for predicting probability accidents. If we compare it with an EEG (Electroencephalogram), which gives us the changes in a wave pattern from one activity to another, but which fails to provide the rate of this shift, while with the above simulator apart from generating a similar wave pattern by TRF, we can additional derive the ROCOF, which can detect this rate of change. Hence, using the pattern of TFR and the ROCOF for predicting the rate would be a great accomplishment.

However, at this stage commenting on the pattern of TFR and ROCOF is premature and may be erroneous. By Table 1, we suggest our hypothetical classification of drivers based on the results of the participant's driving skill in different simulation test courses.

"Table 1. Classification of Drivers". 


\begin{tabular}{|llllll|}
\hline Category & $\begin{array}{l}\text { Speed of Brain } \\
\text { processor } \mathrm{F}_{\mathrm{c}}(\mathrm{Hz})\end{array}$ & ROCOF $_{\mathrm{c}}$ & $\begin{array}{l}\text { Slow driving } \\
\text { compatibility }\end{array}$ & $\begin{array}{l}\text { Fast driving } \\
\text { tendency }\end{array}$ & $\begin{array}{l}\text { Probability of } \\
\text { Collision }\end{array}$ \\
\hline I & $\uparrow$ & $\uparrow$ & +++ & +++ & - \\
\hline II & $\downarrow$ & $\uparrow$ & +++ & + & + \\
\hline III & $\uparrow$ & $\downarrow$ & +++ & ++ & ++ \\
\hline IV & $\downarrow$ & $\downarrow$ & - & - & +++ \\
\hline C critical value, $\uparrow$ increase above or $\downarrow$ decrease below $\mathrm{c}_{,}+++$high,,++ moderate, + low, & - least. \\
\hline
\end{tabular}

The critical values in the Table 1 indicate that the minimum processing speed (or RT) and the ROCOF required by a driver to complete the test simulation with minimum mistakes. Which can be estimated after testing the above simulator on a large population. So that a range of critical processing speed (Reaction time) and the ROCOF, for the different age groups for driving in easy or tough terrain can be determined and used.

Habituation in performing one kind of task can cause a shorter reaction time ${ }^{9}$, however for any new swift in task would require a better ROCOF. Category I indicate the expert drivers who have the shortest reaction time for performing a task and are also quickest in shifting any task. Category IV belongs to the beginner. While category II \& III have median drivers.

Depending on the fact that expertise in performing any activity gives the confidence to perform it faster (determinant of RT) and knowing one's ability to tackle emergency determine the risk-taking behaviour (ROCOF dependent), we hypothesize the fast-driving tendency and the probabilities of accident based on the combination of RT and ROCOF ${ }^{10}$.

Based on the analysis of the table no.1, traffic rules can be formulated and stringently followed. It would also help in monitoring a person's performance by comparing its TRF pattern with the expert. The standardised range of the above parameters can also be used as a marker for fitness in a certain profession like defence skills, aviation, transport, and for deriving a provisional license. This type of stratification can help the researcher classify drivers, which requires further investigation to answer.

Additionally, for making any simulator more humanoid which may garner real-time data, we have the following suggestions-

\subsection{Suggesting additional characteristics in a simulator.}

The current period marks the dawn of technological evolution where 'mixed reality' has come into bloom, giving us an up-close experience of the new technologies. Such immersive technology can be used to design a near-perfect driving simulation. The 4D simulation rides, VR gears, 12D technology (by Voyage Technologies), spatial computer (by Magic leap), and Google Glass (by Google) are just a sneak peek into the colossal orb of this technology. Studies focusing on humans like an advanced driver assistance 
system or the one that involves psychophysical microsimulation models like the Aimsun and VISSIM ${ }^{11}$ and 'form8 driving simulator'12 form a base for more futuristic ventures. A simulator with the capability to analyse various RT components, and the factors affecting them, can yield valuable data. The task so designed for the simulator should have various levels of difficulties that can be made to complete in a stipulated time or at will, simultaneously measuring the rate of occurrence of failure or mistakes.

\subsubsection{Graphics of simulator.}

The simulator's graphics can be designed in the backdrop of the participants' city, overlaid by different terrains. It can be tailor-made for factors relating to the environment, particularly lighting, road surface, and weather conditions.

\subsubsection{Calibration}

The simulator can be well-calibrated in the timing and the threshold of the stimuli, if it coincides with the participants' perception. This estimation helps avoid inaccurate determination of the start time and stop time of stimuli and their response. Furthermore, incorporating maximum modalities of stimuli for calibration and testing could produce a near driving experience. Paradigmatically, for a visual stimulus, timing and threshold can be standardised as per the individual's field of vision by a simplified test like the confrontation test.

\subsubsection{Controls and sensors.}

The response to any stimuli is in the form of either manoeuvring of steering, brake, or pedal. Therefore, any movement in either must be considered for RT. For instance, if in a condition that would require manoeuvring the steering as an essential move rather than braking, then relying on braking to derive RT would be erroneous. If a rider averts a conflict by manoeuvring the steering or grip instead of braking, it should be preferentially counted as the initial response. Therefore, the system should be able to sense a permissible movement of either to calculate the RT.

\subsubsection{Report of the simulation.}

The simulation test analysis should be concluded by a detailed report depicting the routine the speed attained, the number of mistakes committed, time taken to complete the simulation, safe distance kept, acceleration/deceleration used, and additionally should have the ROCOF as per different conditions and terrain. Moreover, the report should contain the rectification suggested by the simulator for the abovementioned parameters. This frequency distribution of the activities of brain can yield valuable information like some pattern of brain activity or help in calculating the fluctuations in ROCOF.

\section{Conclusion}

To have a more humanoid driving simulator we have proposed that it should be well calibrated for individual participant's perception; has the graphics of the participant's familiar terrain; has a sensitivity to detect any manoeuvre in the form of braking, steering or acceleration for obtaining RT. Moreover, it 
should be capable of recording the reaction time of rapidly successive tasks. A reciprocal representation of each RT over the time course of its recording can yield TFD. Based on it, ROCOF can be calculated, and classification of drivers can be postulated. This opens a new way of looking at the brain's activity with multiple outcomes and its interpretations.

\section{List Of Abbreviations}

1. $\mathrm{RT}=$ Reaction time

2. $T F D=$ Time frequency distribution

3. $T F R=$ Time frequency representation

4. $\mathrm{CPU}=$ Central processing unit

5. ROCOF= Rate of change of brain's frequency

\section{Declarations}

\section{Authors' contributions}

PR conceptualized, devised and worked out all the technical details of the project. PR and SC carried out the implementation and performed the analysis under the supervision of PP. The original draft was done by PR. The writing, reviewing and editing was done by PR, LP, after consultation with other authors. The resources were managed by PR \& LP. All authors read and approved the final manuscript.

\section{Competing Interests}

The authors declare that they have no competing interests.

\section{Availability of data and materials}

Data sharing is not applicable to this article, as no datasets were generated or analysed during the current study.

\section{Funding}

The authors did not receive support from any organization for the submitted work.

\section{References}

1. Salthouse, T. A. R Reaction Time. 2, 377-380 (1996). 
2. Oswal, A., Ogden, M. \& Carpenter, R. H. S. The time course of stimulus expectation in a saccadic decision task. J. Neurophysiol. 97, (2007).

3. Lock Down Response Test. http://physicsiology.com/.

4. Akbari, A. \& Haghighi, F. Traffic calming measures: An evaluation of four low-cost TCMs' effect on driving speed and lateral distance. IATSS Res. 44, (2020).

5. Ariën, C. et al. A simulator study on the impact of traffic calming measures in urban areas on driving behavior and workload. Accid. Anal. Prev. 61, (2013).

6. Schneider, D. W. \& Logan, G. D. Modeling task switching without switching tasks: A short-term priming account of explicitly cued performance. Journal of Experimental Psychology: General vol. 134 (2005).

7. S., M. Task switching. Trends Cogn Sci 7, 134-140 (2003).

8. Nagarajan, N. \& Stevens, C. F. How does the speed of thought compare for brains and digital computers? Current Biology vol. 18 (2008).

9. Welford, A. T. Note on the effects of practice on reaction times. J. Mot. Behav. 18, 343-345 (1986).

10. Woodman, T., Akehurst, S., Hardy, L. \& Beattie, S. Self-confidence and performance: A little self-doubt helps. Psychol. Sport Exerc. 11, 467-470 (2010).

11. Astarita, V., Festa, D. C., Giofrè, V. P. \& Guido, G. Surrogate Safety Measures from Traffic Simulation Models a Comparison of different Models for Intersection Safety Evaluation. in Transportation Research Procedia vol. 37 (2019).

12. FORUM8-3D VR \& Visual Interactive Simulation. https://www.forum8.com/.

\section{Figures}

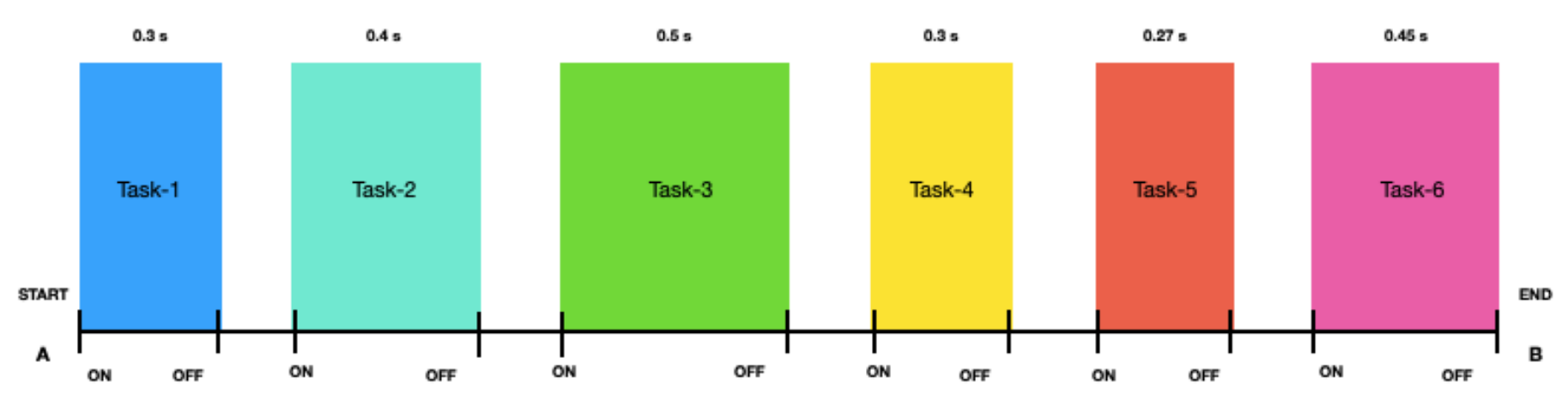

\section{Figure 1}


Switching pattern. Example. Suppose a driving simulator consist of an event of driving a vehicle from point $A$ to point $B$, which consist of multiple tasks like task1- manoeuvrings of a steering after onscreen appearance of turn, task2- tackling speed breaker and so on (as depicted by different colour for each task). The simulator records these rapidly successive tasks. Hence.

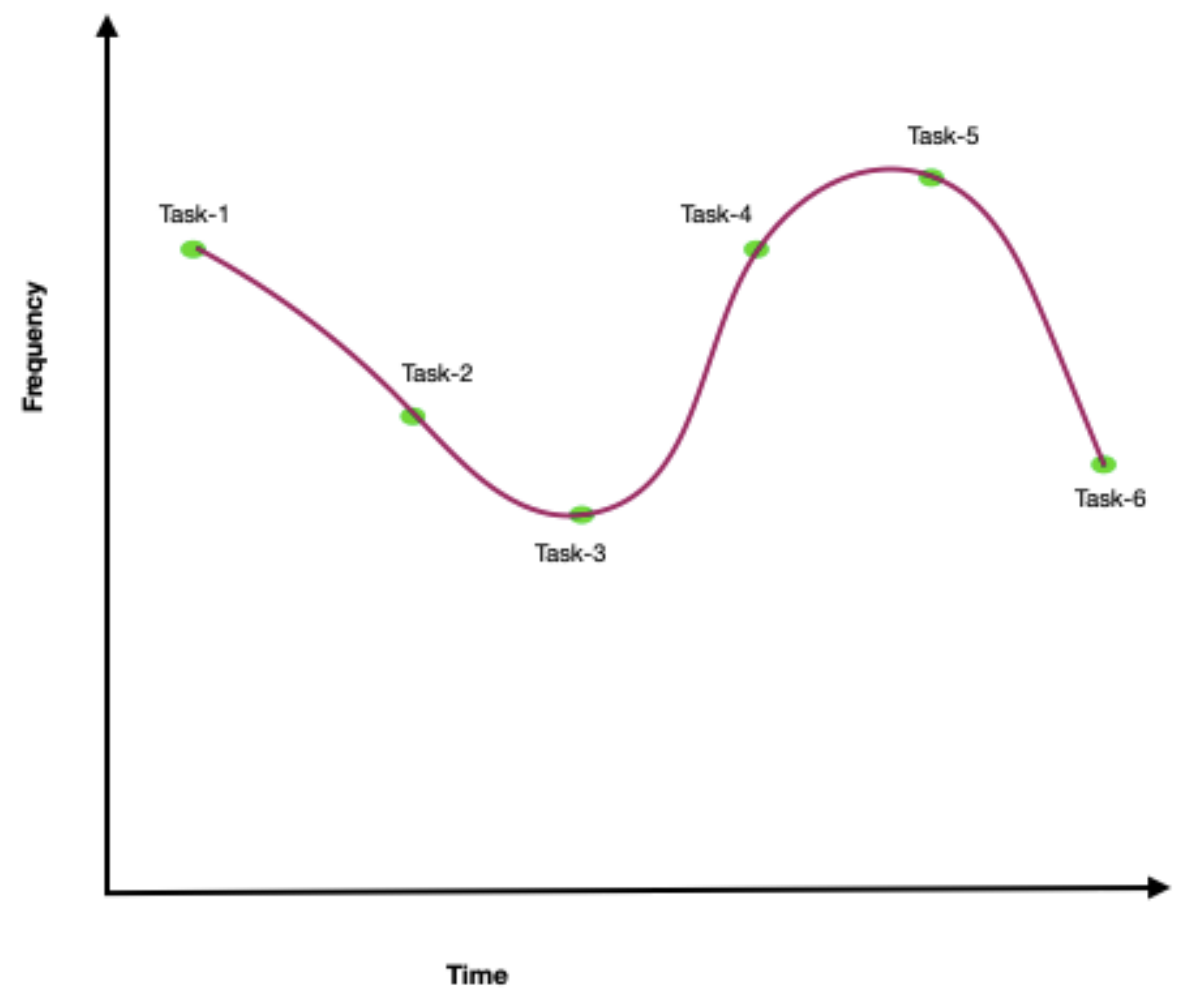

Figure 2

Time Frequency Representation (TFR) 